http://dx.doi.org/10.35381/racji.v5i1.611

\title{
Derecho a opinar y ser escuchado de niños, niñas y adolescentes. Aplicación en justicia especializada
}

\section{Right to comment and be heard of children and adolescents. Application in specialized justice}

\author{
Samantha del Rocío Merchán-Castillo \\ samantha.merchan@ucacue.edu.ec \\ Universidad Católica de Cuenca, Cuenca \\ Ecuador \\ https://orcid.org/0000-0001-6004-9701 \\ Juan Carlos Erazo-Álvarez \\ jcerazo@ucacue.edu.ec \\ Universidad Católica de Cuenca, Cuenca \\ Ecuador \\ https://orcid.org/0000-0001-6480-2270 \\ Camilo Emanuel Pinos-Jaén \\ cpinosj@ucacue.edu.ec \\ Universidad Católica de Cuenca, Cuenca \\ Ecuador \\ https://orcid.org/0000-0002-0934-8471 \\ Cecilia Ivonne Narváez-Zurita \\ inarvaez@ucacue.edu.ec \\ Universidad Católica de Cuenca, Cuenca \\ Ecuador \\ https://orcid.org/0000-0002-7437-9880
}

Recibido: 14 de noviembre de 2019

Aprobado: 15 de diciembre de 2019 


\title{
RESUMEN
}

Se analiza desde la perspectiva del Derechos Constitucional, los errores en los fallos judiciales por la indebida interpretación del Art. 45 de la Constitución, acorde a la evolución del Derecho de Familia se sigue la tendencia de cobijar las garantías de este grupo de atención prioritaria como sujeto de derechos humanos. La metodología empleada es cualitativa, sustentada en métodos de análisis y síntesis bibliográficos. La problemática judicial que acaece radica fundamentalmente en que nuestra ley; no adopta en lo orgánico y procedimental mecanismos para que este derecho a ser consultado sea productivo para la argumentación judicial posterior; por lo que el objetivo es crear un protocolo de acompañamiento, interrogantes y abordaje anexo al código orgánico de la niñez y la adolescencia que garantice este derecho constitucional se efectivice y no se considere un requisito vacío a cumplir dentro de un trámite judicial.

Descriptores: Corte constitucional; Libertad de Expresión; Seguridad Jurídica; Convención Americana de Derechos Humanos, Doctrina de Protección Integral.

\begin{abstract}
It is analyzed from the perspective of Constitutional Rights, errors in judicial decisions due to the improper interpretation of Art. 45 of the Constitution, according to the evolution of Family Law, the tendency to cover the guarantees of this priority attention group is followed as Human rights subject. The methodology used is qualitative, based on bibliographic analysis and synthesis methods. The judicial problem that occurs is fundamentally that our law; does not adopt in the organic and procedural mechanisms so that this right to be consulted is productive for subsequent judicial argumentation; Therefore, the objective is to create an accompanying protocol, questions and approach annexed to the organic code of children and adolescents that guarantees this constitutional right is made effective and is not considered an empty requirement to be fulfilled within a judicial process.
\end{abstract}

Descriptors: Constitutional Court; Freedom of Expression; Legal Security; American Human Rights Convention, Integral Protection Doctrine. 


\section{INTRODUCCIÓN}

La Convención considera esta garantía como una directriz sui generis, como una forma de inter relación personal-procesal, es decir los considera personas a quienes se debe tutelar principalmente dentro de un trámite judicial, pues son seres auto suficientes que, pueden formarse un juicio propio, pese a su edad, frente a determinada circunstancia de su vida, que se encuentra en disputa en una contienda judicial, que muchos de ellos inclusive son entre sus propios progenitores o personas cercanas, como es la familia ampliada. Opinar no es otra cosa que, expresar una opinión de palabra o por escrito, es formarse o tener una idea, juicio o concepto sobre alguien o algo.

¿Frente a los conceptos vertidos, los niños, niñas y adolescentes cuentan con la capacidad para expresarse, tener idea o juicio propio, pese a su edad? La respuesta es sencilla, Si. Pero, asumir este cambio de paradigma surgió cuando se consideró al niño, niña y adolescente (NNA) no como un objeto de protección; por el contrario, se conceptualizó su estatus a sujeto de derechos, obteniendo como resultado que los Estados adopten en sus legislaciones parámetros para adecuar su ordenamiento jurídico interno a esta nueva conceptualización.

Se les reconoce como personas con derechos autónomos de sus progenitores, pues cuentan con capacidades para ejercerlos por sí mismos, lo único que se les pide a los padres, es facilitar este espacio a su favor. Esta concepción de NNA obliga a considerar su interés superior como norma interpretativa, pues cuenta con autonomía progresiva de sus derechos, adquiriendo capacidad propia para ser escuchado, en asuntos de afectación directa a su vida privada.

Dentro del estado ecuatoriano, la Constitución vigente, aprobada mediante referéndum, el día 28 de septiembre del 2008, consagra ciertos principios a favor de este grupo de protección especial, concretamente lo encontramos en el capítulo tercero, sección quinta:

Art. 44.- El Estado, la sociedad y la familia de forma prioritaria deben procurar el desarrollo integral de las niñas, niños y adolescentes, y asegurar ipso facto el ejercicio pleno de sus derechos; se debe atenderá al 
principio de su interés superior por cuanto sus derechos por mandato de la constitución son prevalentes frente a los demás que incluyen sus progenitores. (Asamblea Nacional Constituyente, 2008, art. 44).

Por su parte como eje argumentativo precisamos que los niños tienen per se los derechos a todo ser humano, además algunos específicos en relación a su grado de edad incluso de madurez el estado ecuatoriano reconoce y garantiza inter alias su cuidado y protección desde la concepción, tutela derechos de protección en general v.g derecho a su identidad, nombre y ciudadanía;(..), en líneas generales fundamentalmente se les reconoce una protección aditiva el ser consultados en los asuntos que les afecten; salvo que fuera perjudicial para su bienestar. El Estado garantizará su libertad de expresión y asociación, el funcionamiento libre de los consejos estudiantiles y demás formas asociativas" (Asamblea Nacional Constituyente, 2008, art.45).

Con lo expresado se apertura un nuevo horizonte de interpretación de las garantías a la infancia y adolescencia, pues su interacción con su medio familiar, social cultural y otros merece trato diferenciado. En esta línea de argumentos cuando este derecho hay que aplicarlo en el desarrollo de un trámite judicial, la falencia radica en que no se adopta los mecanismos apropiados para que su opinión pueda ser viabilizada a su favor, obteniendo un resultado de indebida aplicación e interpretación de esta disposición constitucional (Tamayo Vásquez \& Pino Loza, 2019).

En la practica la carencia de protocolos y métodos, convierte este derecho en un formalismo a cumplir dentro de un juicio, lo convierte en un requisito, pues a diario dentro de los espacios judiciales se crean relaciones de subordinación del infante, frente a los padres, familiares, amigos e inclusive de los abogados, ya que lejos del equilibrio entre un dialogo abierto y comprensivo surge una imposición no captada por el Juzgador, quien simplemente sienta una razón que se le escucho, sin analizar y profundizar sobre la decisión a tomar, dentro del litigio judicial, basta con mirar esa interferencia parental al manipular su opinión so pretexto de cumplir con una orden judicial. 
Este trabajo investigativo abordará la correcta aplicación del derecho a ser escuchado de los niños, niñas y adolescentes en justicia especializada, pues la actual problemática judicial radica fundamentalmente en que nuestra ley; no adopta en lo orgánico y procedimental mecanismos para que este derecho a ser consultado sea productivo para la argumentación judicial posterior; pues en la práctica cuando un juez o jueza escucha a un NNA y todo el torrente probatorio dice lo contrario a su opinión, necesariamente debe prevalecer su derecho constitucional a ser consultado por lo tanto el objetivo es crear un protocolo de acompañamiento, interrogantes y abordaje anexo al código orgánico de la niñez y la adolescencia que garantice este derecho constitucional se efectivice y no se considere un requisito vacío a cumplir dentro de un trámite judicial.

\section{DESARROLLO}

\section{Niños, Niñas y Adolescentes, Sujetos De Derecho}

En el génesis de la doctrina de protección integral se estudia y analiza al niño en su condición de sujeto de derechos y mediante la teoría denominada del biopoder que fue considerada y extensamente estudiada por el Psicólogo Michel Foucault, explica la manera de ejercer el poder sobre las personas, la cual ha sido variante por el transcurso del tiempo, recordemos que antes bastaba con enviarlos a la guillotina para someterlos, en la actualidad depende de la cualidad que poseen los gobernantes para manipular y controlar la vida de sus administrados, un ejemplo claro de esto es como se manejan las tácticas de guerra hoy en día, son impartidas por su comandante en jefe, quien sugestiona a sus combatientes que su lucha tenga un objetivo claro, salvar vidas, de toda una nación y no para conservar el poder del Rey como la concepción anterior; en resumen hoy no se busca la muerte sino administrar la vida ajena (Foucalt, 1976).

Este enfoque donde se evidencia gradación de poder, es generalmente aplicado en su contra; principalmente cuando se ven involucrados en problemas judiciales que sus padres crean para tratar de solucionar un problema que ellos no han alcanzado, sin 
considerar el grado de sufrimiento de sus hijos hasta lograr sus objetivos personales, que muchas de las veces únicamente consiste en destruir a su ex pareja; es por este motivo que la Norma normarum, dedica especial protección aquellas personas que por su condición no pueden elevar su voz, denominándolos como sujetos de especial protección constitucional.

Creando a su favor acciones positivas para lograr una igualdad real y efectiva, es decir un agregado de trato diferenciado considerando su edad. En el marco constitucional ecuatoriano se le denomina como grupos vulnerables de atención prioritaria, constantes en los Art. 44, 45 y 35, que en su parte pertinente determina que los ciudadanos, en adultez, personas no emancipadas, mujeres en estado de gestación, en general ciudadanos con capacidades diferentes, privadas de su libertad, y quienes adolezcan de enfermedades catastróficas o de alta complejidad, recibirán atención preferente y especial en lo público y privado. (..) . El Estado prestará especial protección a las personas en condición de doble vulnerabilidad" (Asamblea Nacional Constituyente, 2008).

En este camino a la transformación, por el que atravesó el Ecuador con el nacimiento de la Carta Magna en ciudad Alfaro, Montecristi, Manabí, surgió una nueva era de derechos y justicia para el país, como lo señala el Art. 1 (Asamblea Nacional Constituyente, 2008). Como conocemos el Ecuador es uno de los países que suscribieron y ratificaron la Convención Americana de los Derechos Humanos (CADH) o "Pacto de San José", Convenio que posee un importante catálogo de derechos fundamentales; uno de aquellos es que toda persona tiene derecho acorde a un plazo razonable a ser escuchada ante autoridad judicial, y dentro de la sustanciación de procesos de orden civil, penal o cualquier otra índole más aún en procesos de infancia, pues obligatorio que se respeten sus derechos naturales como el derecho a ser escuchado, y se considere esta opinión al momento de emitir un fallo (Conferencia Especializada Interamericana sobre Derechos Humanos, 1978). 
El alcance universal conseguido radica en considerarlos como un conjunto humano indivisible y de protección especial, les otorga la oportunidad de emitir criterios, perspectivas y enfoques propios, acordes a su edad y circunstancias (Asamblea General de las Naciones Unidas, 1959).

La Comisión utiliza el término de imprescindible el derecho a expresarse jerarquizándolo al punto que coincida con los demás derechos como son de supervivencia, cuidado, protección, salud, educación, etc. ubicándolo en un punto de considerarlo como un derecho humano reconocido a nivel mundial, que se ha conseguido por un largo proceso histórico de conquistas sociales a favor de la infancia.

El indicar que un niño no puede participar activamente en la toma de sus decisiones seria violentar sus derechos humanos, desencadenando en un asunto de absoluta discriminación al impedirles que se expresen, limitando su derecho humano, pues aquellos deben exigirse de forma individual o colectiva, ante cualquier autoridad, pues todos somos iguales, sin importar ninguna condición humana como la religión, la edad, identidad sexual, etc. que pueda determinarse como desigualdad (Asamblea Nacional Constituyente, 2008).

Con lo expresado supra garantizar este fundamental derecho de expresión, que involucra ser consultado constituye un gran avance procedimental, tanto en lo orgánico, constitucional y supra constitucional al conseguir que toda la comunidad internacional lo rescate en sus pronunciamientos lo considere un derecho, abriendo una puerta para su universalización e internacionalización (Eguiguren Praeli, 1992). La libertad de expresión, es desarrollada ampliamente en derechos humanos; se precisa que nadie puede ser molestado a propósito de sus opiniones, toda persona tiene derecho expresarse con libertad, este derecho comprende la libertad de buscar, recibir y difundir informaciones e ideas de toda índole, sin consideración de fronteras, ya sea oralmente, por escrito o en forma impresa o artística, o por cualquier otro procedimiento de su elección" (Asamblea General de las Naciones Unidas , 1976). 
Por tanto, no debemos preguntarnos si es posible o no que niños pequeños, adolescentes cuenten con derecho para libremente emitir sus opiniones, y estas sean consideradas por las autoridades judiciales o administrativas; frente a la existencia de una doctrina proteccionista a su favor. En otro siglo resultaría una herejía, considerarlo, sin embargo y con gran ventaja en la actualidad este tema no es cuestionable.

Lo interesante y discutible en la actualidad es como aplicarla, pues considerando su edad, la comunicación no siempre es verbal, existen otras formas como la no verbal cuando se trata de niñas o niños de muy corta edad, empleando formas como el dibujo, los juegos, observar sus reacciones y sentimientos manifestados, penosamente dentro del sistema judicial ecuatoriano adolece de muchas falencias, corrigiendo las debilidades del sistema, como la falta de infraestructura, preparación, ausencia de conocimiento en los propios juzgadores en materia especializada al momento de administrar justicia y los demás actores del sistema judicial como auxiliares de justicia, abogados, mediadores, testigos, peritos, que deben cumplir con lo determinado ampliamente en la doctrina de materia especializada, desterrando los rezagos de la antigua doctrina del infante colocado como un objeto de cuidado al niño y no como sujeto de derechos.

El análisis realizado por el Comité; en varias observaciones por ejemplos la numero 12 en el párrafo segundo, se resume en la obligación que en todo procedimiento judicial o administrativo que afecte al niño, se debe pedir su opinión, otorgándole oportunidades para que este hecho se evidencie a su favor, principalmente en conflictos judiciales donde se involucran figuras jurídicas familiares de interés social como custodia, tenencia, adopción etc. En asuntos de violencia intrafamiliar o cualquier otro delito en el que se vea involucrado un menor de edad y su correcto desarrollo integral, en temas administrativos donde se de atención a garantizar su salud, educación, seguridad social, solicitudes de refugio o asilo por problemas migratorios, etc., su opinión es trascendente (Convención sobre los Derechos del Niño, 2009). 


\section{El derecho a ser consultado como principio constitucional}

Son varios las garantías tuteladas al momento de ser consultado un niño niña y adolescente, como su libertad de expresión, prima facie; su libertad de pensamiento, conciencia y religión; derecho a la protección de la vida privada y el derecho a la información, pero de su génesis proteccionista surge el respeto a las opiniones de los niños como un derecho fundamental para garantizar el ejercicio de otros. En todo momento de parte de los estados se debe por imperativo garantizar el derecho de opinión libre, entendiéndose un tema de estricto cumplimiento y no discrecional de parte de los operadores de justicia, consecuentemente los Estados, tienen esta obligación mediante la adopción de medidas, que deslumbren el respeto y ejercicio pleno de derechos (Asamblea General de Naciones Unidas, 1989).

La Convención; generó los pilares más importantes en relación del reconocimiento de los derechos humanos a favor de la niñez en consonancia con la doctrina de protección integral, fue uno de los más grandes esfuerzos para tutelar sus principales derechos; se destacan algunos aspectos fundamentales como la formación de catálogos de derechos vg. derecho de participación, supervivencia, de protección y los de desarrollo (Asamblea General de las Naciones Unidas, 1959).

En relación al catálogo indicado supra aquel es de vital importancia, debiendo ser difundidos, interpretados a su favor, pero principalmente deben ser aplicados en cada caso en los que se vean inmersos la infancia y la juventud, para perfeccionarlos dentro de una administración de justicia especializada, para que su eficacia se plasme en cada uno de sus fallos, que no es otra cosa que decidir sobre su vida, considerándolos como sujetos con los mismos derechos que posee una persona adulta, pero con un plus adicional por su condición al no asistirles autodeterminación.

Ahora bien, la principal dificultad acaece desde dos aristas en la aplicación por parte de los operadores de justicia especializados en niñez y adolescencia que desvalorizan los intereses superiores de los NNA al momento de aplicarla; y en la interpretación que abogados en libre ejercicio le dan con carácter acomodaticio en la defensa de sus 
casos particulares, sin prevalecer sus derechos, sino primando el derecho de sus progenitores que generalmente son los que contratan sus servicios profesionales, pues en la práctica utilizan el principio interpretativo del interés superior, inclusive para justificar el no pago de pensiones alimenticias que obviamente va en detrimento de los invocados.

Con estos argumentos las autoridades judiciales y administrativas están obligados a brindar protección, apoyo y promover un desarrollo integral, principalmente dentro de su proceso de crecimiento, maduración y despliegue de su intelecto y de sus capacidades, potencialidades y aspiraciones, en un entorno familiar, escolar, social y comunitario de afectividad y seguridad (Asamblea Nacional Constituyente, 2008). La tutela nace en la propia administración de justicia, en la necesidad de aplicar en forma adecuada el procedimiento para escuchar a un niño y reflejen una correcta y eficiente decisión vislumbrando su interés superior, atendiendo estas consideraciones su enfoque garantista será hacia el Derecho Constitucional y sus principios, como única guía para la justicia especializada a su favor.

El motivo principal por el cual se los debe escuchar, es por el principio desarrollado en la Convención indicada en énfasis exclusivamente lo ordenado en el artículo doce que precisa que los Estados de manera obligatoria con la normativa interna y externa de cada suscriptor deben garantizar el derecho a ser escuchado a todo niño que pueden formarse sus propias opiniones (Asamblea General de Naciones Unidas, 1989). En esta línea de análisis los niños experimentan dificultades en el desarrollo de este derecho, punto considerado por el Comité de los Derechos del Niño (agrupación creada para lograr eficacia en la aplicación de la Convención de los Derechos del Niño entre los Estados suscriptores), al privilegiar a las niñas y niños más pequeños, niños que pertenecen a grupos marginados, desfavorecidos y en extrema pobreza, quienes deben enfrentar problemas por la sectorización superiores a los infantes de su misma edad (Convención sobre los Derechos del Niño, 2009). 
Si bien el Comité cumple una función de dictar observaciones generales con la finalidad de obtener calidad en su práctica, en el año 2006 se desarrolló un debate general sobre sus derechos sobre todo a ser consultado en los asuntos que los afecten, con la finalidad de estudiar su significado e importancia en consonancia con su articulado, efectuando un barrido completo de las posibles lagunas jurídicas, alcances y repercusiones mundiales, principalmente dentro de los procedimientos judiciales, consecuentemente no basta únicamente con escucharlos a manera de cumplimiento de una norma, sino por el contrario se debe meditar esta opinión al momento de decidir (Convención sobre los Derechos del Niño, 2009).

Surge aquí el debate si la opinión puede ser o no redactada en las resoluciones o sentencias judiciales pues muchos jueces comparten la opinión que la misma está protegida por el principio de reserva, quizá sea únicamente por el nombre adoptado, ya que en el Ecuador se la denomina como Audiencia Reservada, pese a que la norma del artículo 60 del Código aplicable, lo rescata más que un derecho a ser consultado como una garantía en la sustanciación de un litigio(Congreso Nacional , 2003).

Se coloca únicamente una razón dentro del proceso de su cumplimiento, coartando el derecho de intervención de abogados a esta diligencia y sin plasmarlos en las decisiones judiciales, hecho perjudicial al momento de interponer un recurso vertical de apelación, pues muchas de las veces se obliga a escucharlo por segunda ocasión; en realidad existen muchas interrogantes en su línea de aplicación, con variadas incertezas pues no existe uniformidad de criterios dentro de la propia Función Judicial de cómo aplicar este derecho, pues todas las normas se encuentran diseminadas en varios tratados internacionales que no han sido unificados en un cuerpo normativo único (htt1).

Los infantes pueden expresar sus opiniones empero, sin presión de ningún tipo, es más pueden escoger si quiere o no ejercer este derecho para ser escuchados; aquello significa también que el niño no puede ser presionado o dirigido, debe encontrarse libre de influencia externa o presiones de familiares y terceros; ya que es bastante común 
dentro del derecho de familia que previamente sea uno de sus progenitores quien lo predisponga a determinada respuesta; el infante no puede ser constreñido a dar su opinión, pese a exigencias normativas existentes, aun cuando sea un requisito legal (htt1).

Consiguientemente se deben garantizar condiciones propias para que la información fluya considerando el grado de vulnerabilidad que puede estar atravesando, su entorno, evitando siempre el re victimizarlo sobre asuntos que afecten su psicología, evitando tener más entrevistas que las estrictamente necesarias, para evitar re victimizaciones innecesarias y prohibidas. El proceso judicial para que sea consultado debe ser dirigido pues puede causar efectos traumáticos al momento de efectuarlo en forma incorrecta, es por esto que, el ejercicio de este derecho obliga que los actores al momento de concretizar el método de escucha informen al niño de los temas a tratar buscando empatía cuales son las opciones las posibles decisiones que pueden adoptarse y sus consecuencias, pues debe estar informado sobre las condiciones en que se le pedirá que exprese sus opiniones, la información es fundamental, porque es condición imprescindible para que existan decisiones apegada a la seguridad jurídica.

Muchos juzgadores, abogados, piensan que escucharlos es una pérdida de tiempo, un lujo, una diligencia sin importancia, pues ellos ya han plasmado todas las pruebas para que determinado proceso judicial salga a su favor, lo cual se convierte en un yerro, ya que generalmente no deciden sobre sus vidas, sino la vida de sus vástagos, personas incapaces temporalmente, sin embargo, se encuentran bajo su cuidado y protección (Convención sobre los Derechos del Niño, 2009).

Una de las conclusiones alcanzas es que no se puede escucharlo en forma correcta si el entorno el método y su forma de hacerlo no es el correcto, intimida, es hostil el ambiente o existen comportamiento, insensibles e inadecuados ara su edad (Convención sobre los Derechos del Niño, 2009) . 


\section{Parámetros de aplicación del protocolo a ser consultado en infancia y adolescencia.}

Uno de los ejes principales para que se efectivice este derecho, es lograr que la niñez y la adolescencia, reciban toda la información, asesoramiento y acompañamiento especializado previo y necesario para que después pueda expresar su opinión con total libertad y principalmente seguridad. Con la aprobación de la Convención ha existido a nivel nacional notables avances, principalmente con la sustitución del Código de Menores Ecuatoriano dentro del cual no se encontraba plasmado este derecho, siendo este el motivo de la ausencia de un protocolo de aplicabilidad en el territorio ecuatoriano.

Sin embargo, después de plasmar los motivos por los cuales el Juez requiere consultar al NNA, y definida su voluntariedad y aceptación de ejercer este derecho, el caso debe ser remitido a la Oficina Técnica de las Unidades Judiciales de la Niñez y Adolescencia, para que un equipo multidisciplinario aborde previamente el espacio dentro del cual se desarrolla, tenemos pues que se obtendrá información previa y confiable sobre las características evolutivas del adolescente, niña o niño, mediante la entrevista con los padres, con el objetivo de indagar sobre el grado de adaptación familiar, social, escolar, deportiva del intervenido. Consiguiendo en primer lugar la predisposición de sus progenitores, pese al conflicto legal por el cual atraviesan, para que preparen a su hijo para la audiencia reservada, pues mientras más colaboradores se muestren los padres, sus hijos también lo estarán predisponiéndoles a superar sus temores, que les pueda generar visitar el Complejo Judicial y no evidencie una victimización secundaria.

El equipo multidisciplinario entrevistará al niño, para indicarle todos los por menores de aquella audiencia reservada y principalmente sobre los puntos a tratar, sin ingresar ninguna idea o concepto ajeno a la realidad por la que atraviesa o nudo critico judicial.

Posterior a ello debemos fijar una fecha, con día, hora y lugar para la evacuación de la misma, de allí surge el primer gran conflicto detectado después de veinte años de ejercicio profesional, pues los servidores judiciales no fomentan la creación de un 
ambiente adecuado para que el menor de edad se exprese, al contrario, priorizan su comodidad, al desarrollarse generalmente en la Sala de Audiencia o en el despacho del Juez, minimizando la falta de comodidad del niño, sin considerar que uno de los elementos indispensables como son: tratar de eliminar la ansiedad y desconfianza que les genera hablar con una persona completamente desconocida a su entorno, a más de aquello abordar los problemas con la verdad.

Es por ello que sería muy importante utilizar las Salas Lúdicas que se encuentran dispuestas en todos Complejos Judiciales del país, actualmente la utilizan para asuntos limitados. Este espacio es óptimo para que permanezcan al contar con cuadros infantiles, juguetes y decoración con colores que puedan resultar tranquilizantes para ellos, es en este lugar en el que deben ser consultados, es decir incomodando al juzgador, pero priorizando los derechos del justiciable que en este caso son los NNA, logrando con ello una entrevista provechosa con un ambiente amistoso y cómodo; dentro del cual debe existir un número reducido de adultos.

Superado el ambiente se debe capacitar a los judiciales que están relacionados con este abordaje, para que las preguntas no resulten intimidatorias en su condición de adulto frente a su entrevistado que es un niño o un adolescente; pues en primer término se debe considerar la situación por la que ellos atraviesan, ya que generalmente se encuentran inmersos en un mar de sentimientos como perdidas, cambios, incertidumbre y estrés, asociado al conflicto en asuntos de tenencia, divorcio o medidas de protección, involucrados involuntariamente. Es importante conocer previamente de parte del entrevistar qué situación se encuentra el infante o adolescente, que generalmente son procesos de desórdenes conductuales, trastornos emocionales, sensaciones de ira, resentimiento, ansiedad, depresión y culpabilidad; reflejada principalmente en agresividad, desobediencia, disminución del trato social, problemas en la relación con la gente de su misma edad y en las relaciones heterosexuales (Philip A. Cowan, 1991). Con esta preparación conseguiremos información confiable y veraz, considerando su grado de madurez y edad, asunto que deberá ser valorado por el juzgador al momento 
de emitir su fallo, en consonancia a su edad y madurez (Congreso Nacional , 2003); es por esto que muchos juzgadores consideran a los niños menores a tres o cinco años como una situación que justifique la falta de evacuación de este derecho, es importante mencionar que al efectuar el abordaje técnico especializado se debe considerar todos estos aspectos.

Quienes deben iniciar con conceptualizar que es verdad y mentira para él o ella, por lo general se pide que emita conceptos sobre aquello, con el objetivo de obtener la verdad. En un segundo momento se debe pasar a explicar que el entrevistador desconoce completamente sobre la realidad por la que atraviesa, para recabar información. Cuando comience a hablar dejarlo que desarrolle íntegramente sus ideas, quizás allí encontremos la respuesta que necesitamos, sin interrupciones, aunque consideremos que estos detalles no son importantes para el caso o resultan inconexos, utilizando preguntas neutras como ¿y qué sucedió después?, luego le pedimos que nos cuente algún episodio relacionado con el nudo del conflicto con detalles, finalmente le podemos pedir aclaraciones sobre temas no que estén claros, evitando que sean sugestivas, con preguntas cortas y con formaciones verbales simples.

Sin utilizar términos cambiantes o inestables como por ejemplo fecha exacta, lugar, día del mes, nombres completos; que llevan a indudablemente a una confusión. Sin utilizar preguntas cuándo y por qué que está completamente prohibido para niños menores de siete años, sino preguntas abiertas, la misma que debe ser grabada para que pueda ser reproducida en segunda instancia o recursos posteriores para evitar su re victimización mientras dure el proceso judicial, obviamente con la reserva del caso (Debbie Joa, 2004).

Las autoridades involucradas en garantizar este derecho deben recordar a priori que se deberá alentar impulsar y fomentar para que un niño se forme una opinión libre y ofrecer un correcto entorno que permita al adolescente y niño ejercer su derecho a ser escuchado (Convención sobre los Derechos del Niño, 2009, p. 11). Estos procesos son parte de los denominados derechos de participación que forman parte del catálogo de 
la doctrina de protección especial, consagrada en el Art. 175 de la Constitución; y dentro de la cual; el ejercicio de sus derechos no son otra cosa más que un elemento fundamental de estos procesos, pues se trata de procesos inclusivos y no deben ser tomados como actos momentáneos, sino un punto de partida para una nueva concepción de los derechos de la infancia (Convención sobre los Derechos del Niño, 2009, p. 13).

\section{METODOLOGÍA}

El tipo de investigación es cualitativa, se usa los métodos inductivo, deductivo y analítico sintético, las técnicas utilizadas fueron análisis de diferentes textos. El estudio de caso fue abordado en base a la práctica de libre ejercicio profesional en un periodo del 2018-2019, guardando la debida confidencialidad e identidad conforme manda el ordenamiento jurídico, sin difundir información. En el análisis de dos casos prácticos se tomó en consideración las decisiones judiciales de primer y segundo nivel, informes técnicos emitidos por el equipo multidisciplinario de la Función Judicial en su calidad de auxiliares de justicia (Almeida, Erazo, Ormaza y Narváez, 2020).

El análisis fue realizado con observancia a los lineamientos doctrinarios, Constitución y Bloque de Constitucionalidad en materia de derechos de justicia especializada, en particular de la Corte Interamericana de Derechos Humanos y Convención de los Derechos del Niño (Ordoñez, Narváez, Trelles y Erazo, 2020).

\section{APORTES GENERADOS}

El derecho del niño a ser consultado sobre asuntos que le afecten directamente en su vida y entorno social, es parte de sus derechos humanos, considerados fundamentales por precautelar el derecho de participación, vida y no discriminación.

Es un eje fundamental la aplicación de la Convención, para que tome importancia este derecho en forma activa y eficaz, a favor de la niñez; la Comisión no ha querido 
imponer parámetros de ejecución para evitar barreras de aplicación, principalmente por la falta de recursos económicos de muchos países suscribientes.

La falta de un protocolo de aplicación, en la justicia ecuatoriana da como consecuencia que no se tutele el derecho a ser consultado, o en otros casos no se obtengan resultados relevantes en desmedro de sus derechos.

Los jueces antes de entrevistarlos deben considerar su edad y grado de madurez para considerar lo manifestado por ellos, es por esto que resulta indispensable que se to efectúe previa intervención, acompañamiento y preparación de parte de un equipo multidisciplinario que facilite el camino para el éxito en la audiencia reservada.

Los niños, niñas y adolescentes no pueden ser obligados, constreñidos a ejercer este derecho, pues lesionaría su interés superior; si deciden ejercerlo hay que considerar que el lenguaje verbal no es el único existente, también debe considerarse la expresión corporal y en algunos casos un lenguaje no verbal.

Hay que evitar la victimización secundaria o re victimización, pues al ejercer su derecho a ser consultado, resultaría perjudicial, vivenciar el evento traumático como la separación de sus progenitores o causas de abandono de los mismos; sin embargo, esta doctrina se maneja más en temas penales, en la actualidad no existen estudios que evidencien que la convocatoria a más de una audiencia reservada, sea nocivo para su desarrollo integral. Atendiendo estas consideraciones resulta obligatorio escucharlos principalmente en asuntos de tenencia, custodia y medidas de protección.

El derecho de consulta y escucha reviste importancia en todas las causas judiciales o administrativas, pues es imperativo para tutelar su interés superior, ya que su opinión debe ser considera además al momento de pronunciamientos llámense sentencias 0 resoluciones valorando también su edad y grado de madurez, que poseen. 
Iustitia Socialis. Revista Arbitrada de Ciencias Jurídicas.

Año V. Vol. V. №1. Edición Especial. 2020

Hecho el depósito de Ley: FA2016000064

ISSN: 2542-3371

FUNDACIÓN KOINONIA (F.K). Santa Ana de Coro, Venezuela

Samantha del Rocío Merchán-Castillo; Juan Carlos Erazo-Álvarez; Camilo Emanuel Pinos-Jaén; Cecilia Ivonne Narváez-Zurita

El derecho a ser consultado se encuentra determinado en el Capitulo de derechos de participaciòn de los niños, niñas y adolescentes estatuido en el Còdigo de la Niñez y

Adolescencia, el mismo que no esta siendo aplicado en debida forma en justicia especializada.

Esta opiniòn se tendrà en cuenta en la medida de su edad y madurez, dentro de todos los fallos en los que se ven involucrados.

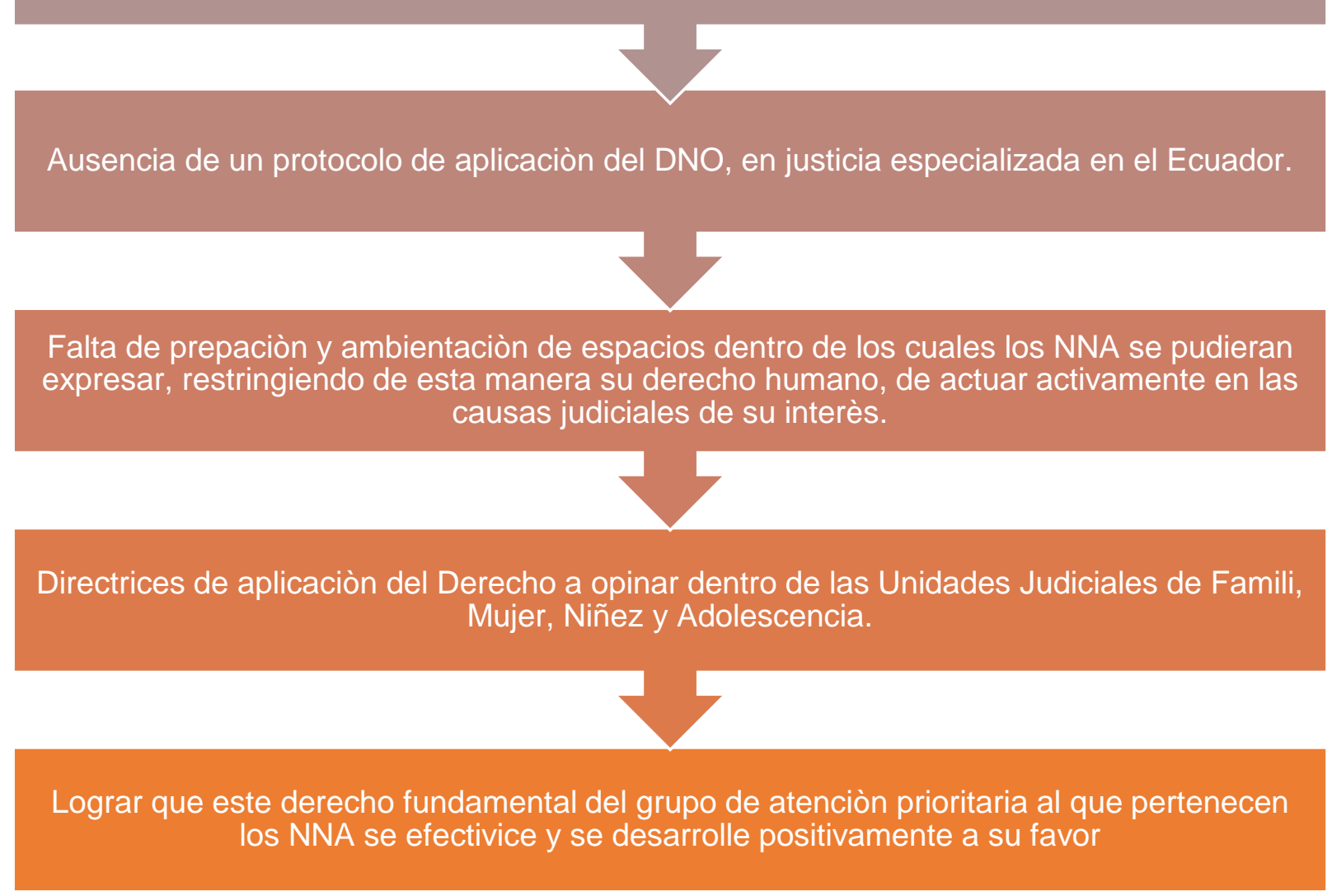

Figura 1 Procedimiento propuesto 


\section{REFERENCIAS CONSULTADAS}

1. Almeida Toral, P. F., Erazo Álvarez, J. C., Ormaza Ávila, D. A., \& Narváez Zurita, C. I. (2020). La aplicación de los derechos humanos en el interés superior del niño. Iustitia Socialis, 639.

2. Asamblea General de las Naciones Unidas . (1976). Pacto Internacional de Derechos Civiles y Politicos. Nueva York, Estados Unidos .

3. Asamblea General de las Naciones Unidas. (1959). Declaración de los derechos del niño.

4. Asamblea General de Naciones Unidas. (1989). Convención de los Derechos del Niño. New York .

5. Asamblea Nacional Constituyente. (20 de octubre de 2008). Constitución de la Republica del Ecuador. Alfaro-Montecristi, Manabi, Ecuador: Registro Oficial 449.

6. Asamblea Nacional Constituyente. (20 de Octubre de 2008). Constitución de la Republica del Ecuador. Alfaro, Montecristi, Manabi, Ecuador: Registro Oficial 449.

7. Bustelo, E. S. (2007). El recreo de la Infancia. Argumentos para otros comienzos. Buenos Aires: Siglo veintiuno.

8. Carretta Muñoz, F. (2018). Algunas precisiones adjetivas sobre el derecho del niño a ser oído, a propósito de un estudio empírico. Revista de derecho (Concepción), 86(243), 93-119. $\quad$ https://dx.doi.org/10.4067/S0718$\underline{591 \times 2018000100093}$

9. Conferencia Especializada Interamericana sobre Derechos Humanos. (11 de Febrero de 1978). Convención Americana sobre Derechos Humanos. Capitulo I. San Jose, Costa Rica : Gacela Oficial Nro. 9460.

10. Congreso Nacional . (2003). Codigo de la Niñez y Adolescencia. Quito, Pichincha, Ecuador: Registro Oficial 737.

11. Convención sobre los Derechos del Niño. (25 de Mayo de 2009). Observación General No. 12. El derecho del niño a ser escuchado . Ginebra.

12. Corte Constitucional de la Republica de Colombia (2011). Sentencia T-167/11. Recuperado de https://www.corteconstitucional.gov.co/relatoria/2011/T-16711.htm 
13. Debbie Joa, M. G. (2004). Resultados legales para niños que han sido abusados sexualmente: impacto de las evaluaciones del Centro de evaluación de abuso infantil.

14. Eguiguren Praeli, F. (1992). ¿Tienen todos los derechos los Derechos Humanos igual jerarquia? IUSET VERITAS.

15. Foucalt, M. (1976). Historia de la sexualidad humana y filosofia. Paris: Gallimard.

16. Ordoñez Andrade, J. I., Narváez Zurita, C. I., Trelles Vicuña, D. F., \& Erazo Álvarez, J. C. (2020). Medida cautelar de suspensión provisional de los servidores y servidoras de la Función Judicial. Iustitia Socialis, 240.

17. Philip A. Cowan, e. M. (1991). Family Transition, Volumen 2.

18. Tamayo Vásquez, F., \& Pino Loza, E. (2019). Derechos Humanos de los niños, niñas y adolescentes en los procesos Arbitrales. IUSTITIA SOCIALIS, 4(7), 97114. doi:http://dx.doi.org/10.35381/racji.v4i7.357

(C2020 por los autores. Este artículo es de acceso abierto y distribuido según los términos y condiciones de la licencia Creative Commons Atribución-NoComercial-Compartirlgual 4.0 Internacional (CC BY-NC-SA 4.0) (https://creativecommons.org/licenses/by-nc-sa/4.0/). 